Meeting report

\title{
Update on developments in the diagnosis and prognostic evaluation of patients with myelodysplastic syndromes (MDS): Consensus statements and report from an expert workshop
}

\author{
Uwe Platzbecker ${ }^{\mathrm{a}, *}$, Valeria Santini ${ }^{\mathrm{b}}$, Ghulam J. Mufti ${ }^{\mathrm{c}}$, Claudia Haferlach $^{\mathrm{d}}$, Jaroslaw P. Maciejewski ${ }^{\mathrm{e}}$, \\ Sophie Park ${ }^{\mathrm{f}}$, Francesc Solég ${ }^{\text {, Arjan A. van de Loosdrecht }}{ }^{\text {, }}$, Detlef Haase ${ }^{\mathrm{i}}$
}

a Universitätsklinikum Carl-Gustav-Carus, Medizinische Klinik I, Dresden 01307, Germany

${ }^{\mathrm{b}}$ AOU Careggi, University of Florence, Italy

${ }^{\mathrm{c}}$ Kings College London and Kings College Hospital, Department of Haematological Medicine, London, UK

d MLL Munich Leukemia Laboratory, Munich, Germany

e Taussig Cancer Institute, Cleveland Clinic, Cleveland, OH, USA

${ }^{\mathrm{f}}$ Hôpital Cochin, Université, Paris V, Assistance Publique des Hopitaux de Paris, Paris, France

${ }^{g}$ Hospital del Mar-Parc de Salut Mar, Barcelona, Spain

h Department of Hematology, VU University Medical Center, Amsterdam, The Netherlands

${ }^{i}$ Department of Hematology and Oncology, Georg-August-University, Göttingen, Germany

\section{A R T I C L E I N F O}

\section{Article history:}

Received 15 September 2011

Received in revised form 7 November 2011

Accepted 8 November 2011

Available online 3 December 2011

\section{Keywords:}

MDS

Diagnosis

Prognosis

\begin{abstract}
A B S T R A C T
Several new treatments for myelodysplastic syndromes (MDS) have recently become available, or are in development. Patients who could benefit from active treatment must be effectively identified and followed up. Therefore, guidelines for the diagnosis and prognostic evaluation of MDS need to be kept up to date with technological and scientific advances. An expert workshop was convened to review currently available and emerging diagnostic technologies and developments in prognostic classification systems, to ensure appropriate management of individual patients. The panel also provided suggestions to ensure adherence to guidelines and highlighted the mandatory requirement for cytogenetic evaluation in patients with MDS.
\end{abstract}

(C) 2011 Elsevier Ltd. All rights reserved.

\section{Introduction}

Myelodysplastic syndromes (MDS) constitute a heterogeneous group of clonal hematopoietic disorders characterized by bone marrow (BM) failure, dysplasia and an increased risk of developing acute myeloid leukemia (AML) [1]. Clinical variability of MDS, diversity of cytomorphological phenotypes and genetic heterogeneity can make the diagnosis and assignment of prognosis a challenge [2]. A number of parameters, both clinical (e.g. cytopenias, age and performance status) and cytological (e.g. blast count, morphology and karyotype) must be taken into account [3,4]. Given the recent development of new treatments that can potentially change the course of the disease [5-8], it is imperative that MDS is correctly diagnosed and classified to ensure appropriate selection and application of treatment. This is particularly important given that inadequate diagnosis can lead to delayed identification, or incorrect classification of, MDS which could result in

\footnotetext{
* Corresponding author. Tel.: +49 351458 4190; fax: +49 3514585362.

E-mail address: Uwe.Platzbecker@uniklinikum-dresden.de (U. Platzbecker).
}

inappropriate treatment decisions [1]. However, many methods applied to patients with suspected MDS, particularly cytogenetics and morphological assessment, are subjective and depend on individual experience. Therefore, strict standardization and assurance of an adequate quality of techniques is required.

As chromosomal aberrations have a huge impact on both the diagnosis and prognosis of patients with MDS, it is essential that accurate cytogenetic analysis is undertaken in patients with MDS. Chromosomal aberrations are present in approximately $50 \%$ of patients with MDS [9], though detection rates seem to vary from institution to institution. This may reflect differences in cohort selection between centers, as the frequency of aberrations is higher in patients with advanced MDS than patients with less advanced disease, and variability in the quality of sample processing between individual laboratories. The impact of cytogenetic status on prognosis, and rate of transformation to AML, is well known and the impact of specific abnormalities is becoming increasingly well understood [10-12]. As an integral component of prognostic scoring systems, including the widely used International Prognostic Scoring System (IPSS) [13], accurate cytogenetic analysis can be instrumental in determining appropriate treatment options from 
best supportive care, immunomodulatory drugs, hypomethylating agents, chemotherapy or hematopoietic stem cell transplantation. Moreover, the selection of certain therapeutic options may be driven by the existence of specific chromosomal abnormalities. For example, lenalidomide is particularly effective in patients with low-risk, transfusion-dependent MDS with a deletion of chromosome $5 q$ [7].

Given the importance of accurate diagnosis of MDS, diagnostic criteria need to be periodically reviewed and updated in order to incorporate modifications in classification criteria, rapid technological developments in diagnostic methodologies, and advances in the understanding of the pathophysiology of the disease. Consequently, an expert panel workshop was convened to review: (i) current guidelines on minimal diagnostic criteria for MDS and obstacles to their implementation; (ii) the role of fluorescence in situ hybridization (FISH), flow cytometry and microarray technologies in improving diagnosis, as well as monitoring of disease progression and assessing response to therapy in patients with MDS; and (iii) how emerging data, particularly cytogenetics, may impact the prognostic classification systems IPSS and World Health Organization (WHO)-based Prognostic Scoring System [WPSS]. The panel also formulated consensus recommendations to ensure inter-laboratory adherence to optimal diagnostic and prognostic procedures.

\section{Current guidelines on minimal diagnostic criteria for MDS and obstacles to their implementation}

Several guidelines regarding the minimal diagnostic criteria for MDS have recently been published.

Following a working conference in 2006 in Vienna, minimal diagnostic criteria [12] were proposed with the following recommendations: two prerequisite criteria: (i) marked and constant cytopenia ( $\geq 6$ months) in at least one cell lineage and (ii) exclusion of another clonal or non-clonal hematopoietic disease or nonhematopoietic disease as the primary reason for cytopenia. At least one out of three additional MDS-related criteria is required: (i) morphological dysplasia in $\geq 10 \%$ of erythroid cells (or $>15 \%$ ringed sideroblasts), neutrophils and their precursors or megakaryocytes in BM smears; (ii) typical cytogenetic abnormality or (iii) a constant blast count of $5-19 \%$. The authors also recommended co-criteria in patients who fulfill both prerequisite criteria and show typical clinical features of MDS, but do not demonstrate any of the three additional criteria. These co-criteria include: (i) an abnormal BM phenotype compatible with a diagnosis of MDS according to European Leukemia Network criteria based on flow cytometry and (ii) clear molecular signs of a monoclonal cell population based on a human androgen receptor assay, gene chip analysis, or mutation analysis (e.g. EZH2 mutations).

Other recently published guidelines, such as the Italian Society of Hematology's practice guidelines, highlight the importance of thorough characterization of patients with suspected MDS for accurate diagnosis and to guide treatment decisions [14]. The guidelines recommend that patients should receive a complete blood count, a peripheral blood (PB) smear with differential leukocyte count, a BM aspiration for cytogenetic and morphologic evaluation, a BM biopsy to assess marrow architecture, cellularity, fibrosis, blast percentage and dysmegakaryopoiesis [15].

Clearly, therefore, guidelines stipulate that cytogenetic analysis of BM should be undertaken in all cases of suspected MDS; at least 20 metaphases should be analyzed [16] and if $<20$ metaphases are scored, then the results should be considered invalid, provided the karyotype appears normal. It is also recommended that patients undergo regular BM analysis to monitor disease progression and response to therapy. FISH analysis, with a validated panel of probes [17], is recommended in certain instances, e.g. to clarify complex aberrations, to detect specific anomalies, e.g. monosomy 7 or $\operatorname{del}(5 q)$, or when only a low number of metaphases are available for standard G-banding $[18,19]$.

Sufficient cytogenetic evaluation is not always undertaken, or is undertaken long after initial presentation, in patients with MDS. One reason for this could be insufficient knowledge among some hematologists and healthcare professionals (HCPs) regarding the importance of cytogenetic analysis for accurate diagnosis, prognosis, and treatment decisions. Importantly, there may also be a lack of inter-laboratory uniformity in cytogenetic methodology. Other reasons for sub-optimal cytogenetic evaluation in patients with suspected MDS include logistical problems, i.e. the time elapsed between BM biopsy/aspirate and analysis and cost issues. Furthermore, BM samples may not always be available. For example, BM aspiration is not always undertaken in elderly patients, or patients with comorbidities, because they are deemed too frail or unsuitable for active treatment options. Although conventional cytogenetic analysis is possible on PB samples, it is frequently unsuccessful or incomplete [20]. Moreover, if it is successful but shows a normal karyotype, the existence of an aberrant clone cannot be excluded because these can sometimes only be detected in BM samples.

\subsection{Recommendations of the expert panel}

The technical challenges of cytogenetic analysis necessitate specialist experience in order for data to be meaningful, and therefore it is recommended that cytogenetic testing be performed by a cytogenetic laboratory experienced in MDS. However, the majority of patients with suspected MDS, especially with mild cytopenias at diagnosis, are seen in community settings which may not have access to an experienced cytogenetics laboratory. These patients should be referred to a hematologist in order to perform the diagnostic work-up. In order to improve karyotyping data across Europe the panel suggested: (i) standardization of the methodology; (ii) increased application of inter-laboratory quality assurance measures which have proved successful in some countries. For example, in the UK a quality assurance system is in place whereby every 3 months test samples are sent to all cytogenetics laboratories; if results are below an acceptable threshold the laboratory is informed and may be inspected by a member of the quality assurance team; and (iii) introduction of a certification process to ensure homogeneity of results across Europe.

The consensus view of the panel was that underdiagnosis is a problem largely due to a poor understanding and awareness of MDS among HCPs. The panel suggested that further education of HCPs and hematologists is required to: (i) improve awareness of MDS, particularly with regard to the importance of undertaking cytogenetic analysis to ensure correct diagnosis and to guide appropriate treatment decisions; (ii) improve knowledge of new treatment options; and (iii) clarify the risks of not undertaking cytogenetic analysis. It is important that HCPs understand that the costs involved in undertaking cytogenetic analysis are far surmounted by therapeutic costs; therefore, avoidance of cytogenetic analysis on cost grounds may be a false economy if it leads to inappropriate therapeutic decisions. With these factors in mind, simple guidelines have been developed to outline criteria required for HCPs to refer cases to hematologists, and to guide hematologists as to what the minimal requirements are from cytogenetic analysis to ensure accurate diagnosis (Table 1). 
Table 1

Recommendations to ensure effective cytogenetic analysis of patients with MDS.

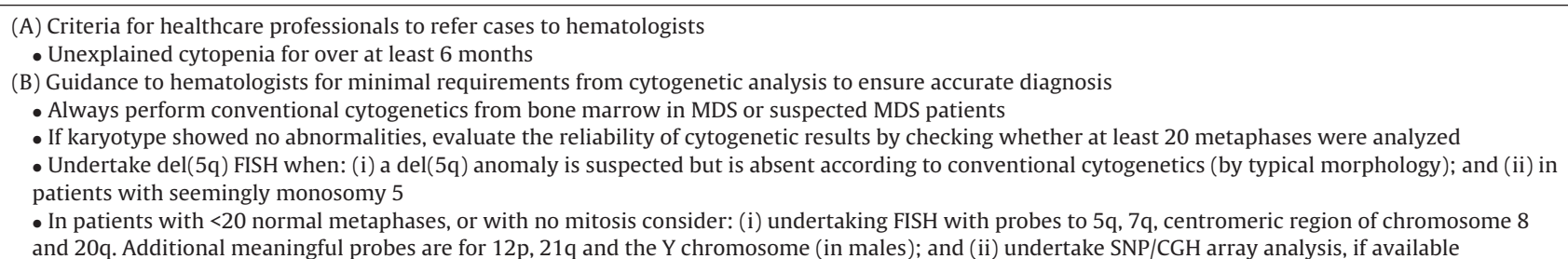

FISH, fluorescence in situ hybridization; MDS, myelodysplastic syndromes.

\section{The role of FISH, flow cytometry and microarray technologies in improving diagnosis, monitoring of disease progression and assessing response to therapy in patients with MDS}

\subsection{FISH}

As FISH analysis does not offer genome-wide coverage and can only be used to evaluate selected chromosomal regions, it is not normally recommended for screening for cytogenetic abnormalities in the first instance. However, in conjunction with conventional cytogenetics FISH could potentially be a powerful approach to improve identification of common chromosomal aberrations in patients with MDS. For example, in a recent study, Mallo et al. analyzed 716 patients who had previously been assessed by standard cytogenetics with a 5q31 FISH probe [19]. Interestingly, 38 (5.9\%) of 637 patients who did not have a detectable del(5q) aberration according to standard cytogenetics had a del(5q) deletion that was detected by FISH only. In these 38 patients, $30 \%$ had no or insufficient $(<20)$ metaphases available for standard cytogenetics.

In another study of 28 patients with MDS or AML, 24 (85.7\%) patients identified as having monosomy 5 with conventional cytogenetics were shown to have $\operatorname{del}(5 q)$ aberrations by FISH, as part of complex aberrations [18]. These findings demonstrate that FISH in addition to conventional cytogenetics may identify additional patients who could benefit from lenalidomide. Consequently, the authors of these studies consider it mandatory to apply 5q31 FISH to all MDS patients with an abnormal karyotype involving chromosome 5 , patients with insufficient metaphases, and patients with a suspected diagnosis of MDS with isolated $5 q$ - where conventional cytogenetics did not reveal del(5q) $[18,19]$.

In addition to providing increased sensitivity for the detection of chromosomal abnormalities, preliminary studies suggest that FISH with a panel of probes could be a useful tool to monitor disease progression and response to therapy, particularly if it could be applied to PB samples. In a recent study where FISH was undertaken on parallel BM and PB samples taken from 77 patients with MDS, concordant results were obtained in $92 \%$ of cases, indicating that PB samples may be informative when following up patients [20]. Braulke et al. recently described a novel method by which circulating CD34+ (hematopoietic progenitor) cells from PB samples were enriched and utilized to monitor response to therapy in 16 patients treated with azacitidine [17]. Analysis of enriched CD34+ cells from PB better reflects the clonal situation in the bone marrow than non-enriched PB. The technique is less invasive than BM aspiration which facilitates frequent monitoring, enabling CD34+ enriched cells to be assessed by FISH once a month immediately prior to each cycle of azacitidine. Cytogenetic response was detected in $67 \%$ of patients using this technique; in each case the cytogenetic response preceded a hematological response by $1-3$ cycles. Despite these encouraging data, another recent study concluded that FISH for $\operatorname{del}(5 q)$ alone may be inadequate for monitoring disease progression and response to therapy, because it may miss cases of cytogenetic progression/clonal evolution [21]
FISH analysis could potentially predict disease progression in patients with active therapy. Braulke et al. demonstrated that in a patient with IPSS high-risk MDS with a complex aberrant karyotype at diagnosis, the complex karyotype was eliminated following eight cycles of azacitidine (only an isolated del[5q] aberration remained). Azacitidine therapy was interrupted between week 32 and week 52; cytogenetic progression was detected at week 52 which preceded loss of hematological improvement that occurred at week 57. More extensive studies are ongoing to investigate utilization of the technique to screen and monitor patients with MDS under different treatment modalities (FISH analysis with a standard [7 probes] or 'super' panel [12 probes] undertaken every 2-3 months, and annually, respectively). Preliminary data have indicated higher than expected occurrences of certain anomalies, e.g. del(12p), and $12 \%$ karyotypic evolution rate within a very short median follow up period of 8 months [22]. FISH could also potentially be used to monitor lower-risk patients receiving supportive care only. The identification of cytogenetic progression could potentially prompt early treatment interventions (e.g. azacitidine or hematopoietic stem cell transplantation) prior to hematological progression thereby improving long-term outcomes.

\subsubsection{Recommendations of the expert panel}

FISH should play a supplementary role to cytogenetics; however, FISH will not supersede conventional chromosomal banding analysis for initial diagnosis of MDS because it may not detect all chromosomal aberrations in a patient [21]. The possibility of frequent FISH analysis on PB samples from the same patient to monitor disease progression and response to therapy is exciting though further prospective data are required in order for this technique to be integrated into standard clinical practice.

\subsection{Flow cytometry}

Cytomorphological analysis is the backbone of the diagnosis and classification of MDS. However, accurate assessment of the type and degree of dysplasia and percentage of blasts can be difficult, with a degree of subjectivity and variability between laboratories. Biological markers are often required to improve diagnosis and classification in order to optimize treatment decisions. As the pattern of cell surface antigen expression during hematopoiesis is highly conserved, aberrant cells can be easily identified by flow cytometry. A number of aberrant antigens have been identified as being of major prognostic importance in patients with MDS. For example the presence of $\mathrm{CD} 7$ on $\mathrm{CD} 34+$ myeloid progenitor cells has been shown to be associated with rapid evolution to AML and poor survival [23].

Standardized combinations of antibodies, as well as technical recommendations, have been put forward in order to facilitate the use of flow cytometry in the diagnosis and prognostic evaluation of MDS [24]. A standardized scoring system to quantify immunophenotypic aberrations in the myelomonocytic lineage, the flow cytometric scoring system, has been developed and validated to facilitate the use of flow cytometry in the clinic $[23,25]$ 
Emerging clinical evidence suggests that flow cytometry could have prognostic value in patients with MDS in terms of helping to predict post-transplant outcomes [26], as well as assessing the risk of transfusion dependence and progression to AML in patients with low-/int-1-risk MDS [23]. Flow cytometry may also be a robust approach to identifying patients with refractory anemia with unilineage dysplasia (RCUD) and refractory cytopenia with multilineage dysplasia (RCMD) according to the WHO 2008. These WHO subgroups are particularly difficult to distinguish by morphology but they do have different prognostic outcomes [16]. However, according to current European Leukemia Network guidelines, the role of flow cytometry is not yet established in the standard diagnostic work-up of MDS, but guidelines are due to be updated in 2011.

Flow cytometry could be useful for predicting response to therapy in patients with MDS. A study of 46 patients with low-/int1-risk MDS, demonstrated that incorporation of flow cytometry into a previously validated model to predict response to erythropoietin defined three patient subgroups with 94,17 and $11 \%$ probabilities of response, respectively [27]. Preliminary data suggest that flow cytometry may identify patients who could benefit from azacitidine therapy and could also identify patients who would benefit from continued prolonged treatment with azacitidine having achieved stable disease [28].

\subsubsection{Recommendations of the expert panel}

At present, flow cytometry cannot substitute for morphology, but may add significantly to the diagnosis and prognostic assessment of patients with MDS and should be integrated into clinical practice when possible and appropriate. Further prospective evaluation is required to assess the role of flow cytometry in treatment decisions. There is a need for standardization of flow cytometric techniques in MDS and steps should be put in place to ensure inter-laboratory reproducibility, which is a major focus within the European Leukemia Network.

\subsection{Microarray technologies}

Developments with microarray technology have provided tools to facilitate high-resolution genome-wide analysis which could potentially supplement traditional methodologies such as standard cytogenetics. Such tools include commercially available single nucleotide polymorphism (SNP) arrays. With this technology genomic DNA is digested with restriction endonucleases, amplified, labeled and hybridized to a chip-based platform that contains DNA probes encompassing thousands of SNPs spread across the entire genome (50 K-, $250 \mathrm{~K}-, 500 \mathrm{~K}$ - and $1000 \mathrm{~K}-S N P$ chips are available). Following hybridization a readout is generated that provides genotype information for each SNP and copy number information across the genome [29].

SNP arrays offer a number of advantages over traditional techniques: (i) higher resolution versus standard cytogenetics (small 'cryptic' deletions and duplications can be detected); (ii) no dependency on the availability of live dividing cells and, therefore, they can provide results when routine cytogenetics are not available; and (iii) regions of uniparental disomy (UPD; chromosomal regions where both copies are derived from the same parent) can be detected. The disadvantages of SNP arrays include an inability to detect balanced rearrangements (i.e. translocations and inversions), an inability to distinguish multiple clones and an inability to detect smaller aberrant cell clones $(<15-20 \%$ of a cell population). However, as cytogenetic abnormalities are relatively frequent and mostly unbalanced in patients with MDS, the disease is conducive to SNP array analysis.
SNP arrays can detect large numbers of chromosomal aberrations in patients with MDS that are not detectable by standard cytogenetics. UPD was detected in $46 \%$ of patients, deletions in $10 \%$ of patients and amplifications in $8 \%$ of cases in a cohort of 119 patients with low-risk MDS who had either a normal karyotype or del(5q) by standard cytogenetics [30]. In another study of 175 patients with MDS, MDS/myeloproliferative disorder or secondary AML, chromosomal lesions were detected in up to $80 \%$ of patients [31]. Patients with a normal karyotype based on standard cytogenetics had reduced survival if cryptic abnormalities were present ( 16 versus 39 months; $P=0.002$ ) indicating that cryptic abnormalities may have some prognostic significance [31].

Array technology has been instrumental in identifying genetic lesions that could both improve knowledge of the underlying pathogenesis of MDS and have prognostic significance. The discovery of a common region of UPD spanning chromosome 4q24 in MDS patients led to the identification of mutations in the TET2 gene [32]. Around $19-26 \%$ of patients with MDS, and approximately $50 \%$ with chronic myelomonocytic leukemia (CMML), have mutations in the TET2 gene, although the prognostic significance of these mutations remain uncertain [33,34]. Discovery of microdeletions by array technology has led to the identification of mutations in genes involved in histone methylation, such as EZH2 on chromosome $7 q 36.1$ and $A S X L-1$ on chromosome 20q11 [35,36]. Mutations in $A S X L-1$ are believed to negatively affect prognosis in patients with MDS [37].

Genetic mutations may predict response to therapy. For example, in a cohort of 63 patients treated with azacitidine, mutations in TP53 were identified as an independent prognostic variable negatively influencing overall survival [38]. In patients with del(5q) MDS, mutations in TP53 are thought to be associated with poor prognosis, loss of response to lenalidomide, and disease progression [39]. In another recent study of 86 patients treated with azacitidine, mutations in TET2 predicted response to therapy independently of karyotype [40].

The adoption of gene mutation analysis into clinical practice could be facilitated by 'next-generation' technology that has several advantages over traditional sequencing, including: (i) improved throughput which allows analysis for a large number of patients. For example, TET2 has recently been fully sequenced in 355 patients with MDS and CMML [41]; (ii) improved sensitivity that enables detection of mutations that are present in only $\sim 1 \%$ cells; and (iii) potential use to monitor clonal evolution during treatment. In a recent study of seven patients treated with azacitidine, sequence analysis suggested that changes in clone size correlated with treatment response [38]. Gene expression arrays could also play a role in the future management of patients with MDS. Recent data have suggested that gene expression arrays can be used to define gene 'signatures' that identify patients with high/intermediate/low risk of progressing to AML [42].

\subsubsection{Recommendations of the expert panel}

Technological advances have led to rapid evolution in the understanding of molecular mechanisms underlying the pathogenesis and malignant transformation of MDS. However, at the moment genomic technologies (SNP arrays, expression arrays, next-generation sequencing) cannot be integrated into standard clinical practice due to lack of expertise and prohibitive costs. For the technologies to enter the clinic in the future, groups need to cooperate to standardize the assays and readout, manufacturers need to make the technologies more user-friendly and cheaper, and further prospective data are required to evaluate the effect of particular gene mutations on diagnosis, treatment outcome and prognosis. 


\section{Impact of emerging data on prognostic classification systems for patients with MDS}

Various prognostic systems have been proposed, with the aim of improving the ability to predict survival and progression in patients with MDS; these include the IPSS and WPSS $[13,43]$.

IPSS remains the gold standard for risk stratification in patients with MDS at diagnosis. However, given recent progress in the dissection of the biology of MDS, and the identification of additional prognostic risk factors, it is likely that IPSS may underestimate risk in its current incarnation. For example, IPSS int-1 patients can sometimes be reclassified as high risk according to the WPSS, a time-dependent regression model, based on WHO classification criteria. Unlike IPSS, WPSS provides dynamic prognostic information and incorporates transfusion dependence as an additional risk factor. However, the definition of transfusion dependency is very subjective across countries and among clinicians. Designation of red blood cell transfusion dependency relies heavily on the opinion of the clinician, who has to take into account the age and cardiac comorbidities of the patient. There is also great discrepancy between clinicians on when to transfuse; some clinicians will transfuse patients with hemoglobin levels $<10 \mathrm{~g} / \mathrm{dL}$ while others will transfuse at a $8 \mathrm{~g} / \mathrm{dL}$ threshold [44]. For these reasons, it has recently been proposed that the WPSS is modified using sexspecific hemoglobin thresholds $(8 \mathrm{~g} / \mathrm{dL}$ for women and $9 \mathrm{~g} / \mathrm{dL}$ for men), instead of transfusion requirement [45]. Analysis of a total of 1344 MDS patients with the modified WPSS was able to identify five distinct risk groups with clearly different survivals [45].

Both IPSS and WPSS also require updating based on improvements in knowledge concerning prognostic factors that have occurred since the introduction of IPSS in 1997 [13], particularly the impact of distinct cytogenetic abnormalities. It is also important to acknowledge that WHO classification criteria of MDS have been updated since the WPSS was developed [46]. Important changes include: (i) the addition of RCUD as a distinct category; (ii) patients with refractory cytopenia(s) but lacking diagnostic morphological features can now be considered as having MDS if they have specific MDS-related cytogenetic abnormalities (i.e. unclassifiable MDS [MDS-U]); (iii) the previously defined RCMD with $\geq 15 \%$ ringed sideroblasts category (RCMD-RS) is no longer considered a separate category due to clinical insignificance and is now incorporated into RCMD; and (iv) patients with $2-4 \%$ blasts in PB and $<5 \%$ blasts in $\mathrm{BM}$ are now diagnosed as refractory anemia with excess blasts1 (RAEB-1) if other clinical and laboratory findings of MDS are present. It is hoped that incorporation of new prognostic information may assist selection of appropriate treatment options and will facilitate prediction of response to therapy.

Many clinical trials use the IPSS for differentiation of 'lower' versus 'higher-risk' disease. However, some patients classified as 'lower-risk', particularly those in the IPSS int-1 group, may have high-risk features, e.g. patients with RBC-transfusion dependent RCMD with intermediate karyotype. Consequently, a revised IPSS (IPSS-R) has been proposed which incorporates several changes including an updated system for assessing cytogenetic risk and the consideration of the severity of cytopenias (with thresholds of hemoglobin: $10 \mathrm{~g} / \mathrm{dL}$; absolute neutrophil count: $0.8 \times 10^{9} \mathrm{~L}^{-1}$; platelets: $100 \times 10^{9} \mathrm{~L}^{-1}$ ). In a retrospective analysis of 4417 patients with MDS, The IPSS-R defined five rather than four major prognostic groups for clinical outcomes [47]. The updated cytogenetic scoring system used in the proposed IPSS-R is based on data from an international study that assessed the prognostic impact of 20 cytogenetic subgroups in $\sim 3000$ patients with MDS who were treated with supportive care only [10]. Abnormalities were grouped as normal, single (one abnormality), double (two abnormalities stratified into three subgroups: double including $\operatorname{del}(5 q)$; double including - 7/7q -; any other double) and complex (three or more abnormalities stratified into two groups: three abnormalities; more than three abnormalities). Based on this classification, five prognostic groups were identified with significant discrimination of overall survival (OS) and risk for leukemic transformation across the groups. Recently, the new cytogenetic classification system has been independently validated in a cohort of 631 consecutive patients with de novo MDS [48]. Application of the same cytogenetic scoring system in the Groupe Francophone des Myelodysplasies register (a combination of treated, untreated and censored patients) resulted in discrimination of OS between groups but did not confer the same level of predictivity as in other patient cohorts.

\subsection{Recommendations of the expert panel}

Both the IPSS and WPSS are robust and clinically meaningful tools to estimate the prognosis of a given MDS patient. As most patients are now treated with active therapy that can alter the natural history of MDS, and improve survival, the panel emphasized the importance of having scoring systems that predict both risk and response to treatment. It is essential that factors are identified that can predict response to particular therapies in patients with different prognostic risk scores. Emerging tools such as flow cytometry and SNP arrays will probably be important in this regard. Nevertheless, at this time, pre-diagnosis assessment of patients' risk based on physiological age and cytogenetic data will continue to underpin the management of the disease. Therefore, new scoring systems need to be validated in cohorts of both untreated and treated patients. This may be challenging, as few untreated patients are now available to validate risk assessment.

\section{Concluding remarks and future perspectives}

The emergence of new therapies that can alter the course of MDS has increased the options available for the management of the disease. However, it is essential that patients who could benefit from these treatments are accurately diagnosed as soon as possible following initial presentation. For this to become a reality, it would be desirable for physicians to have access to screening tools that would allow them to monitor disease progression in patients during its early stages and help them to assess when active treatment is appropriate, as well as monitoring the response to therapy.

Moving forward, morphological assessment and standard metaphase cytogenetics will remain central to the diagnosis of MDS. However, FISH could play an important supplementary role, particularly in detecting specific abnormalities (e.g. del[5q]) when insufficient metaphases are available for standard cytogenetics. Recent data have demonstrated that a multifaceted diagnostic approach can help improve diagnostic precision in patients with suspected MDS $[2,49]$. Rapid progress has already been made in the development of methodologies to monitor disease progression and response to therapy. For example, FISH analysis of PB samples is being intensively studied and has shown significant promise. In addition, flow cytometry may prove to be a valuable tool in the diagnosis, prognostic evaluation, and monitoring of response to therapy in patients with MDS and may be integrated into standard clinical practice in the future. Developments in molecular technologies have led to huge strides in the understanding of the molecular pathogenesis of MDS. As our knowledge of genes involved in the pathogenesis and progression of MDS increases, this should have an impact on prognostic scoring systems and allow for better stratification of patients for different types of treatment. As diagnostic and prognostic guidelines develop over the coming years, a major challenge will be to ensure adherence to guidelines across centers in different countries. 


\section{Role of funding source}

Development of this manuscript was supported by funding from Celgene International.

\section{Conflict of interest statement}

UP, DH, VS and $\mathrm{CH}$ have received honoraria from Celgene and Novartis. JM has received research support from Celgene, Eisai, Alexion and honoraria from Celgene and Alexion. SP has received honoraria from Celgene, Amgen and Janssen-Cilag. Other authors disclose no conflicts of interest relevant to the content of this manuscript.

\section{Acknowledgement}

The authors would like to acknowledge writing assistance of Lynn Pritchard of FireKite during the development of this manuscript.

Contributions. All persons listed as co-authors actively participated in the Celgene-convened expert workshop (Munich, 27 October 2010) and contributed equally to the development of panel recommendations. Each co-author provided essential input into the development of the manuscript and approved the final version of the document.

\section{References}

[1] Malcovati L, Nimer SD. Myelodysplastic syndromes: diagnosis and staging. Cancer Control 2008;15(Suppl.):4-13.

[2] Bacher U, Haferlach T, Kern W, Weiss T, Schnittger S, Haferlach C. The impact of cytomorphology, cytogenetics, molecular genetics, and immunophenotyping in a comprehensive diagnostic workup of myelodysplastic syndromes. Cancer 2009;115:4524-32.

[3] Catenacci DV, Schiller GJ. Myelodysplasic syndromes: a comprehensive review. Blood Rev 2005;19:301-19.

[4] Komrokji RS, Bennett JM. Evolving classifications of the myelodysplastic syndromes. Curr Opin Hematol 2007;14:98-105.

[5] Fenaux P, Mufti GJ, Hellstrom-Lindberg E, Santini V, Finelli C, Giagounidis A et al. Efficacy of azacitidine compared with that of conventional care regimens in the treatment of higher-risk myelodysplastic syndromes: a randomised, open-label, phase III study. Lancet Oncol 2009;10:223-32.

[6] Kantarjian H, Issa JP, Rosenfeld CS, Bennett JM, Albitar M, DiPersio J, et al. Decitabine improves patient outcomes in myelodysplastic syndromes: results of a phase III randomized study. Cancer 2006;106:1794-803.

[7] List A, Dewald G, Bennett J, Giagounidis A, Raza A, Feldman E, et al. Lenalidomide in the myelodysplastic syndrome with chromosome $5 q$ deletion. $N$ Engl J Med 2006;355:1456-65.

[8] Sloand EM, Wu CO, Greenberg P, Young N, Barrett J. Factors affecting response and survival in patients with myelodysplasia treated with immunosuppressive therapy. J Clin Oncol 2008;26:2505-11.

[9] Haase D, Germing U, Schanz J, Pfeilstocker M, Nosslinger T, Hildebrandt B, et al New insights into the prognostic impact of the karyotype in MDS and correlation with subtypes: evidence from a core dataset of 2124 patients. Blood 2007;110:4385-95.

[10] Schanz J, Tüchler F, Sole F, Mallo M, Hildebrandt B, Slovak M, et al. Proposal of a new, comprehensive cytogenetic scoring system for primary MDS. Haematologica 2010;95(Suppl. 2):219 [Abstract 0535].

[11] Tricot G, Boogaerts MA, De Wolf-Peeters C, Van den Berghe H, Verwilghen RL. The myelodysplastic syndromes: different evolution patterns based on sequential morphological and cytogenetic investigations. $\mathrm{Br} \mathrm{J}$ Haematol 1985;59:659-70.

[12] Valent P, Horny HP, Bennett JM, Fonatsch C, Germing U, Greenberg P, et al. Definitions and standards in the diagnosis and treatment of the myelodysplastic syndromes: consensus statements and report from a working conference. Leuk Res 2007;31:727-36.

[13] Greenberg P, Cox C, LeBeau MM, Fenaux P, Morel P, Sanz G, et al. International scoring system for evaluating prognosis in myelodysplastic syndromes. Blood 1997;89:2079-88.

[14] Santini V, Alessandrino PE, Angelucci E, Barosi G, Billio A, Di MM, et al. Clinical management of myelodysplastic syndromes: update of SIE, SIES, GITMO practice guidelines. Leuk Res 2010;34:1576-88.

[15] Valent P, Orazi A, Busche G, Schmitt-Graff A, George TI, Sotlar K, et al. Standards and impact of hematopathology in myelodysplastic syndromes (MDS) Oncotarget 2010;1:483-96.
[16] Steidl C, Steffens R, Gassmann W, Hildebrandt B, Hilgers R, Germing U, et al. Adequate cytogenetic examination in myelodysplastic syndromes: analysis of 529 patients. Leuk Res 2005;29:987-93.

[17] Braulke F, Schanz J, Jung K, Shirneshan K, Schulte K, Schuetze C, et al. FISH analysis of circulating CD34+ cells as a new tool for genetic monitoring in MDS: verification of the method and application to 27 MDS patients. Leuk Res 2010;34:1296-301.

[18] Galvan AB, Mallo M, Arenillas L, Salido M, Espinet B, Pedro C, et al. Does monosomy 5 really exist in myelodysplastic syndromes and acute myeloid leukemia? Leuk Res 2010;34:1242-5.

[19] Mallo M, Arenillas L, Espinet B, Salido M, Hernandez JM, Lumbreras E, et al. Fluorescence in situ hybridization improves the detection of $5 q 31$ deletion in myelodysplastic syndromes without cytogenetic evidence of $5 \mathrm{q}-$. Haematologica 2008;93:1001-8.

[20] Cherry AM, Slovak ML, Campbell LJ, Chun K, Eclache V, Haase D, et al. Will a peripheral blood (PB) sample yield the same diagnostic and prognostic cytogenetic data as the concomitant bone marrow (BM) on myelodysplasia? An international study comparing cytogenetics and interphase FISH using parallel PB and BM samples. Blood 2010;116 [Abstract 2922].

[21] Gohring G, Giagounidis A, Busche G, Hofmann W, Kreipe HH, Fenaux $P$, et al. Cytogenetic follow-up by karyotyping and fluorescence in situ hybridization: implications for monitoring patients with myelodysplastic syndrome and deletion 5q treated with lenalidomide. Haematologica 2011;96: 319-22.

[22] Braulke F, Schanz J, Detken S, Seraphin J, Metz M, Brümmerdörf T, et al. Detection of rare abnormalities and karyotype evolution in MDS patients from peripheral blood: first results from The Mulitcenter German Prospective Diagnostic CD34+FISH-Study. Haematologica 2010;95:219 [Abstract 0536].

[23] van de Loosdrecht AA, Westers TM, Westra AH, Drager AM, van der Velden $\mathrm{VH}$, Ossenkoppele GJ. Identification of distinct prognostic subgroups in lowand intermediate-1-risk myelodysplastic syndromes by flow cytometry. Blood 2008:111:1067-77.

[24] van de Loosdrecht AA, Alhan C, Bene MC, la Porta MG, Drager AM, Feuillard J, et al. Standardization of flow cytometry in myelodysplastic syndromes: report from the first European LeukemiaNet working conference on flow cytometry in myelodysplastic syndromes. Haematologica 2009;94:1124-34.

[25] Wells DA, Benesch M, Loken MR, Vallejo C, Myerson D, Leisenring WM, et al. Myeloid and monocytic dyspoiesis as determined by flow cytometric scoring in myelodysplastic syndrome correlates with the IPSS and with outcome after hematopoietic stem cell transplantation. Blood 2003;102:394-403.

[26] Scott BL, Wells DA, Loken MR, Myerson D, Leisenring WM, Deeg HJ. Validation of a flow cytometric scoring system as a prognostic indicator for posttransplantation outcome in patients with myelodysplastic syndrome. Blood 2008;112:2681-6.

[27] Westers TM, Alhan C, Chamuleau ME, van der Vorst MJ, Eeltink C, Ossenkoppele GJ, et al. Aberrant immunophenotype of blasts in myelodysplastic syndromes is a clinically relevant biomarker in predicting response to growth factor treatment. Blood 2010;115:1779-84.

[28] Alhan C, Westers TM, Eeltink C, Cali C, Ossenkoppele GJ, Van de Loosdrecht AA. Flow cytometric score correlates with clinical response to azacitidine in intermediate- 2 and high risk myelodysplastic syndrome patients. Blood 2010;116 [Abstract 441].

[29] Maciejewski JP, Mufti GJ. Whole genome scanning as a cytogenetic tool in hematologic malignancies. Blood 2008;112:965-74.

[30] Mohamedali A, Gaken J, Twine NA, Ingram W, Westwood N, Lea NC, et al. Prevalence and prognostic significance of allelic imbalance by singlenucleotide polymorphism analysis in low-risk myelodysplastic syndromes. Blood 2007; 110:3365-73.

[31] Gondek LP, Tiu R, O'Keefe CL, Sekeres MA, Theil KS, Maciejewski JP. Chromosomal lesions and uniparental disomy detected by SNP arrays in MDS, MDS/MPD, and MDS-derived AML. Blood 2008;111:1534-42.

[32] Mohamedali AM, Smith AE, Gaken J, Lea NC, Mian SA, Westwood NB, et al. Novel TET2 mutations associated with UPD4q24 in myelodysplastic syndrome. J Clin Oncol 2009;27:4002-6.

[33] Delhommeau F, Dupont S, Della VV, James C, Trannoy S, Masse A, et al. Mutation in TET2 in myeloid cancers. N Engl J Med 2009;360:2289-301.

[34] Langemeijer SM, Kuiper RP, Berends M, Knops R, Aslanyan MG, Massop M, et al. Acquired mutations in TET2 are common in myelodysplastic syndromes. Nat Genet 2009;41:838-42.

[35] Gelsi-Boyer V, Trouplin V, Adelaide J, Bonansea J, Cervera N, Carbuccia N, et al. Mutations of polycomb-associated gene ASXL1 in myelodysplastic syndromes and chronic myelomonocytic leukaemia. Br J Haematol 2009;145: $788-800$.

[36] Nikoloski G, Langemeijer SM, Kuiper RP, Knops R, Massop M, Tonnissen ER, et al Somatic mutations of the histone methyltransferase gene EZH2 in myelodysplastic syndromes. Nat Genet 2010;42:665-7.

[37] Garcia-Manero G. Prognosis of myelodysplastic syndromes. Hematology Am Soc Hematol Educ Program 2010 2010:330-7.

[38] Kulasekararaj AG, Mohamedali AM, Smith AE, Lea NC, Kizilors A, Abdallah A, et al. Polycomb complex group gene mutations and their prognostic relevance in 5-azacitidine treated myelodysplastic syndrome patients. Blood 2010;116 [Abstract 125]

[39] Jadersten M, Saft L, Pellagatti A, Gohring G, Wainscoat JS, Boultwood J, et al. Clonal heterogeneity in the 5q-syndrome: 53 expressing progenitors prevail during lenalidomide treatment and expand at disease progression. Haematologica 2009;94:1762-6. 
[40] Itzykson R, Kosmider O, Cluzeau T, Mansat-De MV, Dreyfus F, Beyne-Rauzy O, et al. Impact of TET2 mutations on response rate to azacitidine in myelodysplastic syndromes and low blast count acute myeloid leukemias. Leukemia 2011. April 15 [Epub ahead of print].

[41] Smith AE, Mohamedali AM, Kulasekararaj A, Lim Z, Gaken J, Lea NC, et al. Nextgeneration sequencing of the TET2 gene in 355 MDS and CMML patients reveals low-abundance mutant clones with early origins, but indicates no definite prognostic value. Blood 2010;116:3923-32.

[42] Mills KI, Kohlmann A, Williams PM, Wieczorek L, Liu WM, Li R, et al. Microarraybased classifiers and prognosis models identify subgroups with distinct clinical outcomes and high risk of AML transformation of myelodysplastic syndrome. Blood 2009; 114:1063-72.

[43] Malcovati L, Germing U, Kuendgen A, la Porta MG, Pascutto C, Invernizzi $\mathrm{R}$, et al. Time-dependent prognostic scoring system for predicting survival and leukemic evolution in myelodysplastic syndromes. J Clin Oncol 2007;25:3503-10.

[44] Gale RP, Barosi G, Barbui T, Cervantes F, Dohner K, Dupriez B, et al. What are RBC-transfusion-dependence and -independence? Leuk Res 2011;35: $8-11$.
[45] Malcovati L, la Porta MG, Strupp C, Ambaglio I, Kuendgen A, Nachtkamp K, et al Impact of the degree of anemia on the outcome of patients with myelodysplastic syndrome and its integration into the WHO classification-based Prognostic Scoring System (WPSS). Haematologica 2011. June 9 [Epub ahead of print].

[46] Vardiman JW, Thiele J, Arber DA, Brunning RD, Borowitz MJ, Porwit A, et al The 2008 revision of the World Health Organization (WHO) classification of myeloid neoplasms and acute leukemia: rationale and important changes. Blood 2009;114:937-51.

[47] Greenberg P, Tuechler H, Schanz J, et al. Revised International Prognostic Scoring System (IPSS-R), developed by the International Prognostic Working Group for Prognosis in MDS (IWG-PM). Leuk Res 2011;35:S6.

[48] Bernasconi P, Klersy C, Boni M, Dambruoso I, Zappatore R, Cavigliano P, et al. The International Multicentric Cooperation cytogenetic scoring system effectively predicts disease outcome in de novo MDS. Haematologica 2011;96(S2): 99.

[49] Makishima H, Rataul M, Gondek LP, Huh J, Cook JR, Theil KS, et al. FISH and SNP-A karyotyping in myelodysplastic syndromes: improving cytogenetic detection of $\operatorname{del}(5 q)$, monosomy $7, \operatorname{del}(7 q)$, trisomy 8 and $\operatorname{del}(20 q)$. Leuk Res 2010;34:447-53. 